\title{
How to Interpret a Functional or Motility Test - Colon Transit Study
}

\author{
Eun Ran Kim and Poong-Lyul Rhee*
}

Department of Medicine, Samsung Medical Center, Sungkyunkwan University School of Medicine, Seoul, Korea

Measurement of colon transit time is the most basic and primary tool in evaluating disorders of colonic motility. In particular, it is helpful in pathologic diagnosis and for planning management in patients with constipation. Several techniques for measuring colon transit time currently exist. The standard measurement of colon transit time has been performed using radioopaque marker test. The radioopaque marker test is the most widely used method; it is simple to perform as well as being cost effective. But, this technique produces radiation exposure. Radionuclide scintigraphy and wireless motility capsules are other techniques used to measure colon transit time. In radionuclide scintigraphy, the transit of radioisotope is viewed by gamma camera; this approach has an advantage in that it uses minimal radiation and it allows a physiological assessment of gastrointestinal transit. Wireless motility capsules have been validated most recently, but this technique is not useful in Korea. This review presents the techniques used to measure colon transit time and the interpretations provided in different colon transit studies.

(J Neurogastroenterol Motil 2012;18:94-99)

\section{Key Words}

Colon transit time; Colonic scintigraphy; Radioopaque markers; Wireless motility capsule

\section{Introduction}

Colonic motility is a critical process underlying the major functions of the large bowel such as storage, absorption, propulsion and defecation. ${ }^{1}$ Disorders of colonic motility typically present with constipation or diarrhea. Measurement of colonic transit time is useful in evaluating colonic motility, and allows both the severity of the problem and the response to therapy to be assessed.

Different methods exist to investigate colon transit time. The standard measurement of colonic transit time has been performed with radioopaque markers or colonic scintigraphy. The traditional approach is to assess the progression time of radioopaque markers along the large bowel. ${ }^{2}$ Colonic scintigraphy can evaluate whole-gut transit. Recently, wireless motility capsules have also been validated as a technique in measuring colon transit time.

\section{Radioopaque Markers}

Assessment of colon transit based on ingested radioopaque markers has been widely adopted since Hinton et $\mathrm{al}^{3}$ first described this technique in 1969. Radioopaque marker testing distinguishes constipation subgroup such as normal or slow transit

Received: December 16, 2011 Revised: December 30, 2011 Accepted: December 30, 2011

(c) This is an Open Access article distributed under the terms of the Creative Commons Attribution Non-Commercial License (http://creativecommons. org/licenses/by-nc/3.0) which permits unrestricted non-commercial use, distribution, and reproduction in any medium, provided the original work is properly cited.

*Correspondence: Poong-Lyul Rhee, MD

Division of Gastroenterology, Department of Medicine, Samsung Medical Center, Sungkyunkwan University School of Medicine, 50 Irwon-dong, Gangnam-gu, Seoul 135-710, Korea

Financial support: None.

Tel: +82-2-3410-3409, Fax: +82-2-3410-6983, E-mail: plrhee@skku.edu

Conflicts of interest: None. 
constipation, and assesses segmental transit times in patients with delayed total colon transit. ${ }^{4}$ This test is simple and inexpensive as well as reliable and reproducible. However it requires good compliance of the patient, produces radiation exposure, and does not measure the transit of a physiological meal.

\section{Methods}

Radioopaque markers are plastic beads or rings that are usually ingested in a capsule containing 20-50 markers. Two kind of radioopaue markers, Sitzmarks ${ }^{\circledR}$ (Konsyl Pharmaceuticals, Texas, USA) and Kolomark ${ }^{\mathrm{TM}}$ (M.I.Tech., Pyongtaik, Korea) ${ }^{7,8}$ is commonly used in Korea. Each single capsule contains 24 or 20 radioopaque markers, respectively.

Several methods have been suggested when it comes to using radioopaque markers, including the single capsule technique and the multiple capsules technique. ${ }^{9,10}$ The single capsule technique requires ingestion of markers in a single capsule on a specific day, followed by several abdominal X-rays that are repeated until all markers are defecated or a single abdominal X-ray on day 5 (120 hours later). However the "single ingestion with multiple X-ray" technique is time-consuming and produces greater radiation exposure. The multiple capsules technique requires the ingestion of 1 capsule a day for 3 days, followed by abdominal X-rays on day 4 and 7 or only on day 7 .

\section{Interpretation}

Interpretation is based on the identification of markers in 3 regions namely the right, left and rectosigmoid regions. These are defined by bony landmarks and gaseous outlines as described by Arhan et $\mathrm{al}^{11}$ (Fig. 1).

In the single capsule technique with a single abdominal X-ray on day 5 (120 hours later), delayed transit is defined as $>20 \%$ re- tention of markers. Evans et $\mathrm{al}^{12}$ measured the time taken for radioopaque markers to pass through the large bowel in 25 healthy men and 18 healthy women. They demonstrated that $95 \%$ of normal subjects pass more than $80 \%$ of markers within 120 hours.

In the multiple capsule technique, colon transit times in each segment and through the entire colon are calculated by multiplying the number of markers by 1.2 (or by 1.0 when using a capsule containing 24 markers). ${ }^{13}$

Normative data for colon transit time in adults are available from a large number of radioopaque markers studies. ${ }^{10,14-17}$ In most such studies from western, the mean colon transit time was

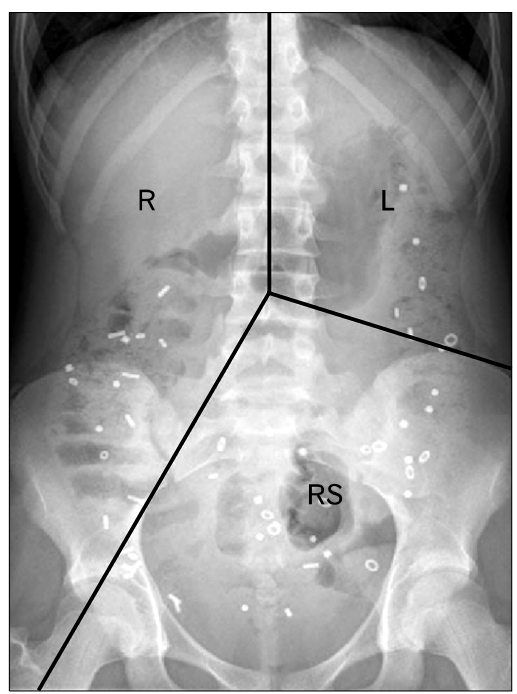

Figuure 1. Colonic segments in abdominal X-ray. Spinal processes and imaginary lines from fifth lumbar vertebra to right pelvic outlet and left iliac crest, serving as landmarks defined projection zones of right, left and rectosigmoid colon. $\mathrm{R}$, right colon; L, left colon; RS, rectosigmoid colon.

Table. Colon Transit Times Measured Using Radioopaque Markers in Healthy Korean Adults 7,18,20-22,24

\begin{tabular}{|c|c|c|c|c|c|c|c|}
\hline \multirow{2}{*}{ Author } & \multirow{2}{*}{$\begin{array}{l}\text { X-ray } \\
\text { on day }\end{array}$} & \multirow{2}{*}{ Number } & \multirow{2}{*}{$\begin{array}{l}\text { Age (mean } \pm \mathrm{SD} \\
\text { or range, yr) }\end{array}$} & \multicolumn{4}{|c|}{ Colon transit time (mean $\pm \mathrm{SD}$ or median [range], hr) } \\
\hline & & & & Total colon & Right colon & Left colon & RS colon \\
\hline Koo et al, ${ }^{22} 1991$ & 4 & 28 & & $22.4 \pm 4.9$ & $8.7 \pm 4.2$ & $9.8 \pm 4.3$ & $3.9 \pm 4.8$ \\
\hline Bak et al, ${ }^{21} 1996$ & 7 & $32(\mathrm{~m}=5)$ & $40-54$ & $24(1.7-32.4)$ & $4.8(1.2-7.2)$ & $3.6(0.0-20.4)$ & $0.0(0.0-7.2)$ \\
\hline Kim et al, 2001 & 4,7 & $30(\mathrm{~m}=15)$ & $43.3 \pm 13.2$ & $30.3 \pm 14.9$ & & & \\
\hline Lee $^{24} 2010$ & 4,7 & $75(\mathrm{~m}=35)$ & $41.9 \pm 13.7$ & $20.5 \pm 20.3$ & $5.4 \pm 7.2$ & $7.6 \pm 11.0$ & $7.7 \pm 12.1$ \\
\hline Kim et $\mathrm{al}^{20} 2003$ & 4,7 & $15(\mathrm{~m}=11)$ & $50.2 \pm 1.5$ & $24.0 \pm 4.1$ & $6.88 \pm 1.17$ & $10.80 \pm 2.59$ & $4.96 \pm 1.19$ \\
\hline \multirow[t]{3}{*}{ Jung et al, ${ }^{18} 2003$} & 4,7 & 42 & $34 \pm 7$ & $26.5 \pm 19.4$ & $5.9 \pm 6.9$ & $9.2 \pm 9.2$ & $11.5 \pm 11.1$ \\
\hline & & $\mathrm{m}=21$ & $35 \pm 7$ & $22.3 \pm 16.1$ & $5.9 \pm 7.7$ & $6.0 \pm 6.1$ & $5.9 \pm 7.7$ \\
\hline & & $f=21$ & $34 \pm 8$ & $30.1 \pm 21.4$ & $5.8 \pm 6.2$ & $12.5 \pm 10.7$ & $5.7 \pm 6.2$ \\
\hline
\end{tabular}

RS, rectosigmoid; m, male; f, female. 
30-40 hours, with upper normal limit of 70 hours in mixed populations. However, there were differences in colon transit times between studies due to differences in age, gender ratio, race and methodology. Women had a longer maximal colon transit time compared with men. ${ }^{4,15,16,18}$ In women, menstral cycle influenced colon transit times. ${ }^{15,18}$

Also, colon transit times are generally shorter in normal Asian populations than in Westerners. In studies from Korea, mean colon transit time was 20-30 hours in normal subjects (Table). ${ }^{7,-22}$ Moreover, Chan et $\mathrm{al}^{23}$ found that the colon transit time was $24.5 \pm 18.8$ hours (21 hours in men and 28 hours in women) in healthy Chinese adults. Despite the current lack of a direct comparative study of Asian and Westerners, dietary differences may make a difference in colon transit time between Asian and Westerner. ${ }^{24}$ Consumption of fiber or spicy foods may be higher in Asian compared to Western populations.

\section{Colonic Transit Scintigraphy}

The utilization of a radionuclide to measure gut transit was first demonstrated by Krevsky et $\mathrm{al}^{25}$ in 1986 using cecal intubation. The scintigraphic technique use minimal radiation and has been shown to be a reliable alternative for accurate quantitative measurement of colonic transit. In addition, the test offers reproducible and accurate performance across a spectrum of common colonic motility disorders, linking colonic transit measurements to biological processes, and provides correct prediction of outcomes in therapeutic interventions. ${ }^{26}$ Therefore, this test is in- dicated to measure whole-gut and regional colonic transit in patients with suspected colonic motility disorders or more diffuse disorders involving the stomach and small bowel. ${ }^{4}$

On the other hand, scintigraphy studies are very long in duration making them impractical for many nuclear medicine departments. $^{6}$

\section{Methods}

Scintigraphy transit tests involve the ingestion of radioactive isotopes, where their progression through the gastrointestinal tract is followed with a large-field-view gamma camera (Fig. 2). The test uses the long half-life radionuclides ${ }^{111}$ indium. There are 2 methods for the delivery of markers in clinical uses: ${ }^{111}$ indiumdiethylenetriamine pentaacetic acid $\left({ }^{111}\right.$ In-DTPA) labeled water consumed in a standard solid-liquid meal ${ }^{27,28}$ and ${ }^{111}$ indium/activated charcoal slurry contained within a $\mathrm{pH}$-sensitive polymer methacrylate- coated capsule designed to break down in the alkaline environment of terminal ileum and release the radioisotope into the lumen. ${ }^{9,29}$ A shorter half-life isotope, technetium $99 \mathrm{~m}$, can be added to measure gastric and small bowel transit. In practice, one should image the colon at 24 and 48 hours to identify patterns of transit different from normal. ${ }^{30}$

\section{Interpretation}

Studies use many different reporting modes including transit time in hours $\left(T_{1 / 2}\right)$, percentage of radioactivity retained, proximal colonic emptying or center of mass. ${ }^{10}$

Generally, 2 end points are used to summarize colonic trans-
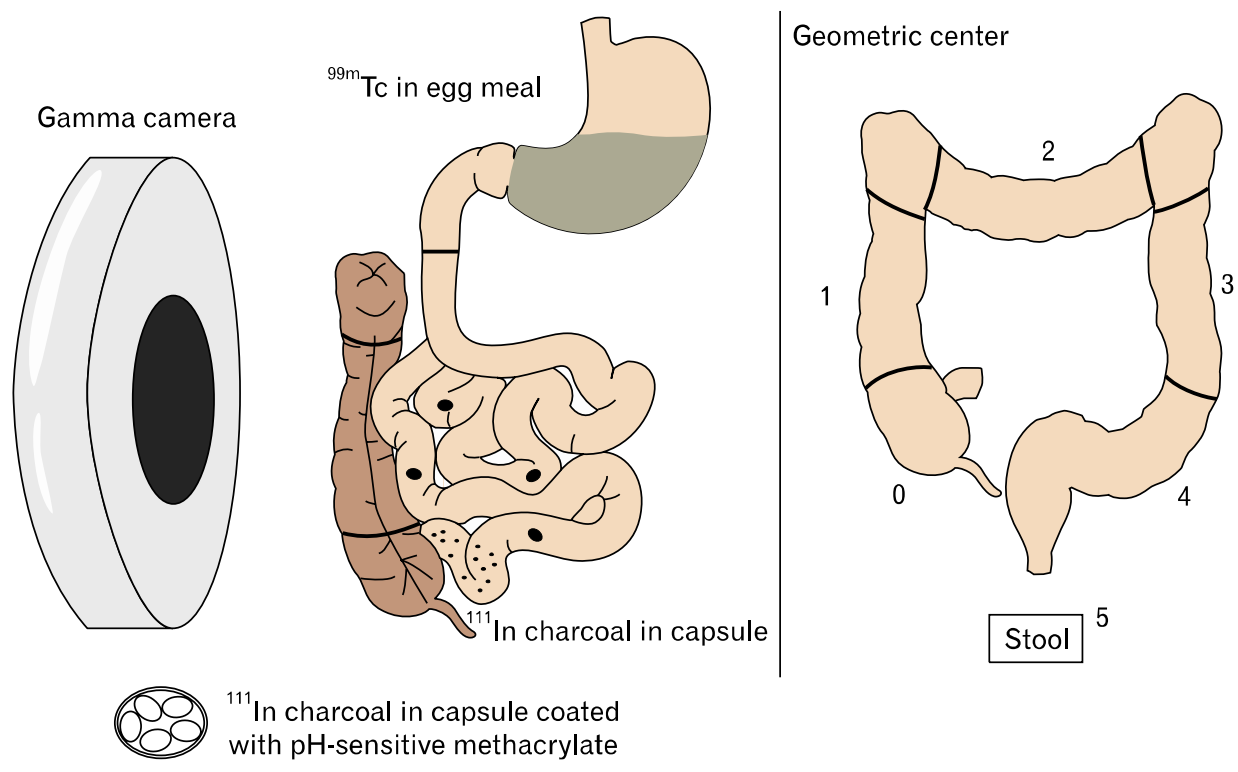


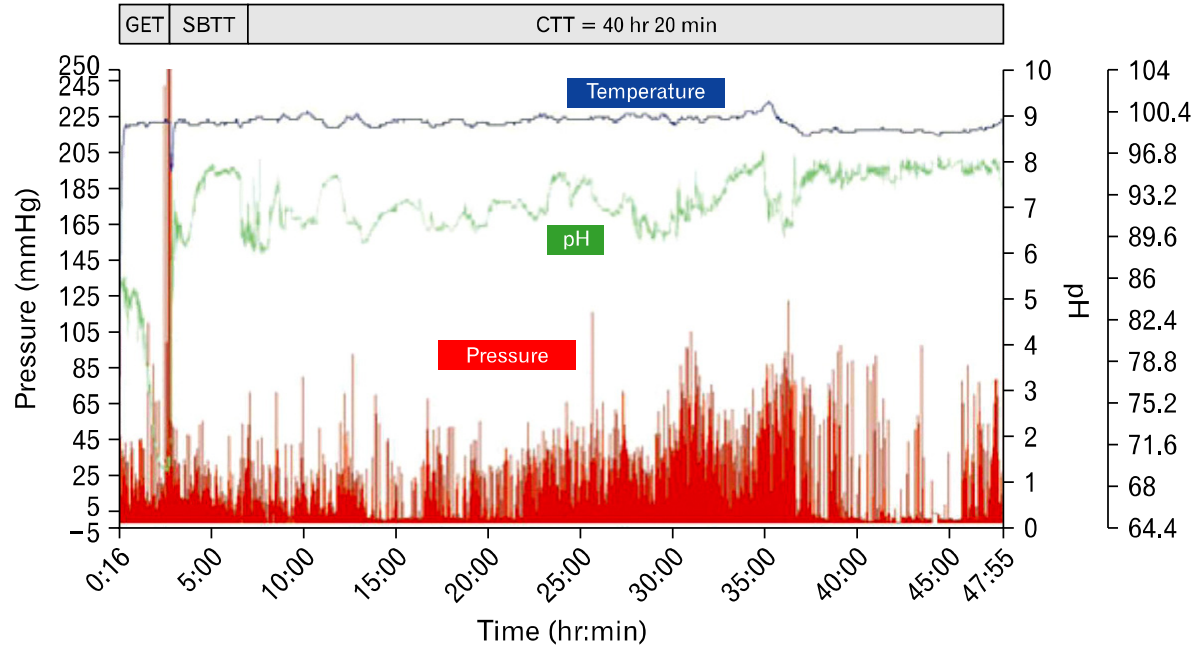

Figure 3. Wireless motility capsule: this figure displays wireless motility capsule profiles from healthy subject who had normal colon transit time as well as normal gastric emptying time. GET, gastric emptying time; SBTT, small bowel transit time; CTT, colon transit time. it: (1) overall colon transit, expressed as geometric center; and (2) emptying of the ascending colon. ${ }^{4,26}$

The colon can be divided into 5 or 7 regions of interest. The 5 regions-of-interest program is commonly used to quantitate counts in each colonic segment: ascending colon (AC), transverse colon (TC), descending colon (DC) and rectosigmoid (RS), numbered as segments 1-4, respectively. Segment 5 refers to expelled stool (S). ${ }^{4,26,31}$

The geometric center (GC) is the weighted average of the isotope distribution within colon and stool. The GC is expressed as the sum of the multiplication of the proportion of ${ }^{111}$ In counts in each colonic segment at a given time by that segment's weighting factor ${ }^{32}$ :

$$
\begin{aligned}
\mathrm{GC}= & {[(\% \mathrm{AC} \times 1)+(\% \mathrm{TC} \times 2)+(\% \mathrm{DC} \times 3)+(\% \mathrm{RS}} \\
& \times 4)+(\% \mathrm{~S} \times 5)] / 100
\end{aligned}
$$

A high GC implies fast colonic transit because the center of the activity has progressed to the left side of the colon or has been eliminated in the stool, whereas a low GC implies slow colonic transit because the center of the activity is in the proximal colon. $^{26,32}$

Maurer et $\mathrm{al}^{30}$ determined that using solid-liquid meal, the normal mean $( \pm 1 \mathrm{SD}) \mathrm{GC}$ values were $4.6 \pm 1.5$ at 24 hours and $6.1 \pm 1.0$ at 48 hours in a recent update based on their experience and previous studies. ${ }^{10,33}$

The emptying of the $\mathrm{AC}$ is summarized as the $\mathrm{T}_{1 / 2}$ (time for $50 \%$ emptying) as calculated by linear interpolation of values on the AC emptying curve. ${ }^{26}$ The measurement of proximal colon emptying with GC can be useful in the differentiation of patients with slow colonic transit and pelvic outlet obstruction. ${ }^{34}$

\section{Wireless Motility Capsule}

A wireless motility capsule system (SmartPill GI Monitoring System, The SmartPill Corporation, Buffalo, NY, USA) was approved by the Food and Drug Administration in 2006 for the assessment of gastric emptying and whole gut transit time. ${ }^{35}$

The wireless motility capsule (WMC) test is well tolerated, exhibit good compliance and measures colon transit time without radiation exposure. ${ }^{4}$ Therefore, the WMC test has potential to be a useful diagnostic test to evaluate patients for gastrointestinal transit disorders and to study for colonic responsiveness to pharmacologic agents.

However, although a few studies showed a moderately strong correlation between the WMC test and radioopaque markers study for colon transit, ${ }^{36}$ there is not enough evidences about the availability of the WMC test for measuring colon transit time, currently. In addition, it is expensive, requires physician training for interpretation and device failure is reported in $\sim 3 \%$ of cases. ${ }^{4}$ Above all, the WMC test is not available in Korea.

\section{Methods}

The WMC system consists of a wireless ingestible motility capsule, a portable data receiver worn by the patients for acquiring data, and data analysis software (MotiliGI; SmartPill Corporation, Buffalo, NY, USA). The WMC system houses sensors for $\mathrm{pH}$, temperature and pressure, and has the ability to transmit the sensed data at $434 \mathrm{MHz}$. The shape and dimensions of the WMC system (cylindrical, $26.8 \mathrm{~mm}$ long by $13 \mathrm{~mm}$ in diameter) are similar to those of pill endoscopy. The system's MotiliGI software provides calculations and graphical displays of the data 
(Fig. 3). Data can be reviewed in real time or after study completion. ${ }^{6,36}$ The WMC system's battery lasts for at least 5 days after activation. ${ }^{6}$ The capsule measures $\mathrm{pH}$ (range, 0.05-9.00 $\mathrm{pH}$ units), pressure (range, $0-350 \mathrm{mmHg}$ ) and temperature (range, $\left.25-49^{\circ} \mathrm{C}\right) .^{36}$

\section{Interpretation}

Whole gut transit is defined as the interval between capsule ingestion and its exit from the body. ${ }^{6}$ The capsule exit time is verified by a loss of signal and/or abrupt drop in temperature.

Capsule gastric emptying time is defined as the duration of the time from WMC ingestion to pyloric passage, as determined by an abrupt $\geq 2 \mathrm{pH}$ unit increase from the lowest postprandial value to at least 4 that does not decrease below 4 for $>10 \mathrm{mi}-$ nutes at any subsequent time. ${ }^{37,38}$ Small bowel transit time is defined as the time interval between capsule entry into the small bowel and its entry into the cecum. The cecal entry is defined as a distinct drop of more than $1 \mathrm{pH}$ unit that is sustained for more than 10 minutes and occurs at least 30 minutes after capsule entry into the small bowel. ${ }^{37,39}$

Delayed gastric emptying is diagnosed when the emptying time exceeds 5 hours (range: 2 to 5 hours). Delayed small intestinal transit is determined when transit times exceed 6 hours (range: 2 to 6 hours), based on 95\% cutoff values from control studies. Delayed colon transit is diagnosed when transit times exceed 59 hours (range: 10 to 59 hours). ${ }^{36-39}$

\section{Conclusion}

Assessment of colon transit is important in patients with symptoms of colonic dysmotility because it can provide useful mechanistic insights and demonstrate response to treatment. Until now, a variety tests have been available for measuring colon transit. Radioopaque marker tests have traditionally been performed to measure colon transit time and this technique is the most widely used method to date. Recently, the colonic scintigraphy and the WMC test have been validated for measuring whole gut transit as well as colon transit. However, the colonic scintigraphy is used less frequently in clinical practice and the WMC test is not available in Korea.

Therefore, radioopaque marker test is clinically useful for the detection of altered colon transit in Korea. It is also important to perform the colon transit study considering clinical purpose and patient's condition.

\section{References}

1. Wald A. Motility disorders of the colon and rectum. Curr Opin Gastroenterol 2012;28:52-56.

2. Pomerri F, Frigo AC, Grigoletto F, Dodi G, Muzzio PC. Error count of radiopaque markers in colonic segmental transit time study. AJR Am J Roentgenol 2007;189:W56-W59.

3. Hinton JM, Lennard-Jones JE, Young AC. A new method for studying gut transit times using radioopaque markers. Gut 1969;10: 842- 847 .

4. Rao SS, Camilleri M, Hasler WL, et al. Evaluation of gastrointestinal transit in clinical practice: position paper of the American and European Neurogastroenterology and Motility Societies. Neurogastroenterol Motil 2011;23:8-23.

5. Bouchoucha M, Prado J, Chtourou L, Devroede G, Atanassiu C, Benamouzig R. Non-compliance does not impair qualitative evaluation of colonic transit time. Neurogastroenterol Motil 2011;23:103108.

6. Maqbool S, Parkman HP, Friedenberg FK. Wireless capsule motility: comparison of the SmartPill GI monitoring system with scintigraphy for measuring whole gut transit. Dig Dis Sci 2009;54:21672174.

7. Kim JE, Rhee PL, Kim YH, et al. [Clinical usefulness of KolomarkTM, a Korean radio-opaque marker for measuring colon transit time.] Korean J Med 2001;60:337-341. [Korean]

8. Kim JE, Rhee PL, Kim YH, et al. [The Development of Kolomark(TM), A Korean radio-opaque marker for measuring colon transit time.] Korean J Gastrointest Motil 1999;5:136-139. [Korean]

9. Dinning PG, Di Lorenzo C. Colonic dysmotility in constipation. Best Pract Res Clin Gastroenterol 2011;25:89-101.

10. Southwell BR, Clarke MC, Sutcliffe J, Hutson JM. Colonic transit studies: normal values for adults and children with comparison of radiological and scintigraphic methods. Pediatr Surg Int 2009;25:559572.

11. Arhan P, Devroede G, Jehannin B, et al. Segmental colonic transit time. Dis Colon Rectum 1981;24:625-629.

12. Evans RC, Kamm MA, Hinton JM, Lennard-Jones JE. The normal range and a simple diagram for recording whole gut transit time. Int $\mathrm{J}$ Colorectal Dis 1992;7:15-17.

13. Chaussade S, Khyari A, Roche H, et al. Determination of total and segmental colonic transit time in constipated patients. Results in 91 patients with a new simplified method. Dig Dis Sci 1989;34:11681172.

14. Martelli H, Devroede G, Arhan P, Duguay C. Mechanisms of idiopathic constipation: outlet obstruction. Gastroenterology 1978;75: 623-631.

15. Meir R, Beglinger C, Dederding JP, et al. [Age- and sex-specific standard values of colonic transit time in healthy subjects.] Schweiz Med Wochenschr 1992;122:940-943. [German]

16. Metcalf AM, Phillips SF, Zinsmeister AR, MacCarty RL, Beart RW, Wolff BG. Simplified assessment of segmental colonic transit. Gastroenterology 1987;92:40-47.

17. Sadik R, Abrahamsson H, Stotzer PO. Gender differences in gut 
transit shown with a newly developed radiological procedure. Scand J Gastroenterol 2003;38:36-42.

18. Jung HK, Kim DY, Moon IH. Effects of Gender and Menstrual Cycle on Colonic Transit Time in Healthy Subjects. Korean J Intern Med 2003;18:181-186.

19. Suh SW, Park HJ, Jung HY, et al. [Comparison of 4 - day 7 - day methods in the evaluation of colon transit time.] Korean J Gastroenterol 2001;38:241-246. [Korean]

20. Kim YH, Sim SG, Cho KR, et al. [Colonic transit time in patient with liver cirrhosis.] Korean J Gastroenterol 2003;42:394-399. [Korean]

21. Bak YT, Kim JH, Lee CH. Cisapride in chronic idiopathic constipation: clinical response and effect on colonic transit time. Korean J Intern Med 1996;11:151-156.

22. Koo C, Kim MJ, Sung JH, et al. Colonic transit time in irritable bowel syndrome. Korean J Intern Med 1991;41:324-331.

23. Chan YK, Kwan AC, Yuen H, et al. Normal colon transit time in healthy Chinese adults in Hong Kong. J Gastroenterol Hepatol 2004;19:1270-1275.

24. Lee OY. Asian motility studies in irritable bowel syndrome. J Neurogastroenterol Motil 2010;16:120-130.

25. Krevsky B, Malmud LS, D'Ercole F, Maurer AH, Fisher RS. Colonic transit scintigraphy. A physiologic approach to the quantitative measurement of colonic transit in humans. Gastroenterology 1986;91:1102-1112.

26. Camilleri M. Scintigraphic biomarkers for colonic dysmotility. Clin Pharmacol Ther 2010;87:748-753.

27. Bonapace ES, Maurer AH, Davidoff S, Krevsky B, Fisher RS, Parkman HP. Whole gut transit scintigraphy in the clinical evaluation of patients with upper and lower gastrointestinal symptoms. Am J Gastroenterol 2000;95:2838-2847.

28. Maurer AH, Krevsky B. Whole-gut transit scintigraphy in the evaluation of small-bowel and colon transit disorders. Semin Nucl Med 1995;25:326-338.

29. Burton DD, Camilleri M, Mullan BP, Forstrom LA, Hung JC.
Colonic transit scintigraphy labeled activated charcoal compared with ion exchange pellets. J Nucl Med 1997;38:1807-1810.

30. Maurer AH, Parkman HP. Update on gastrointestinal scintigraphy. Semin Nucl Med 2006;36:110-118.

31. Stivland T, Camilleri M, Vassallo M, et al. Scintigraphic measurement of regional gut transit in idiopathic constipation. Gastroenterology 1991;101:107-115.

32. Deiteren A, Camilleri M, Bharucha AE, et al. Performance characteristics of scintigraphic colon transit measurement in health and irritable bowel syndrome and relationship to bowel functions. Neurogastroenterol Motil 2010;22:415-423, e95.

33. Krevsky B, Maurer AH, Niewiarowski T, Cohen S. Effect of verapamil on human intestinal transit. Dig Dis Sci 1992;37:919-924.

34. Eising EG, von der Ohe MR. Differentiation of prolonged colonic transit using scintigraphy with indium-111-labeled polystyrene pellets. J Nucl Med 1998;39:1062-1066.

35. Sarosiek I, Selover KH, Katz LA, et al. The assessment of regional gut transit times in healthy controls and patients with gastroparesis using wireless motility technology. Aliment Pharmacol Ther 2010; 31:313-322.

36. Rao SS, Kuo B, McCallum RW, et al. Investigation of colonic and whole-gut transit with wireless motility capsule and radiopaque markers in constipation. Clin Gastroenterol Hepatol 2009;7:537544.

37. Kuo B, Maneerattanaporn M, Lee AA, et al. Generalized transit delay on wireless motility capsule testing in patients with clinical suspicion of gastroparesis, small intestinal dysmotility, or slow transit constipation. Dig Dis Sci 2011;56:2928-2938.

38. Kuo B, McCallum RW, Koch KL, et al. Comparison of gastric emptying of a nondigestible capsule to a radio-labelled meal in healthy and gastroparetic subjects. Aliment Pharmacol Ther 2008;27:186196.

39. Rao SS, Mysore K, Attaluri A, Valestin J. Diagnostic utility of wireless motility capsule in gastrointestinal dysmotility. J Clin Gastroenterol 2011;45:684-690. 